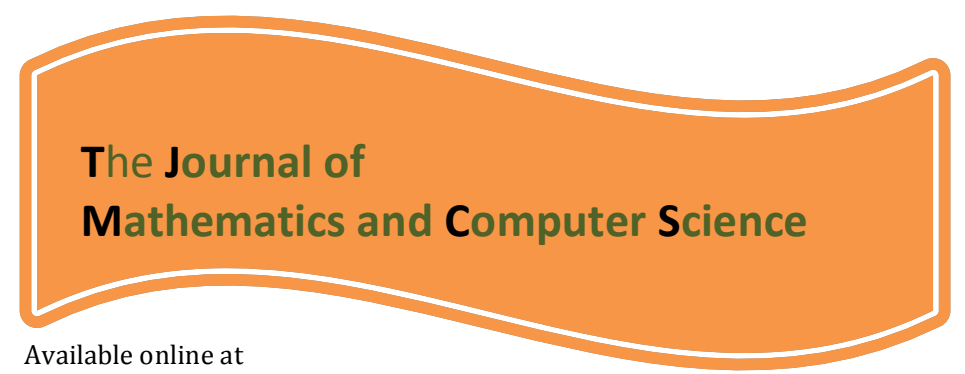

http://www.TIMCS.com

The Journal of Mathematics and Computer Science Vol .3 No.2 (2011) 225 - 235

\title{
Numerical solution for Maxwell's equation in metamaterials by Homotopy Analysis Method
}

\author{
A.Zare ${ }^{1}$, M.A.Firoozjaee ${ }^{2}$ \\ 1. Malek Ashtar University of Technology \\ 2. Department of Mathematics, Shahid Beheshti University, Tehran, Iran \\ mrc@amut-es.ac.ir \\ M64arab@math.com
}

Received: May 2011, Revised: June 2011

Online Publication: December 2011

\begin{abstract}
In this paper, the Homotopy analysis Method (HAM) is applied to the Maxwell system. The HAM yields an analytical solution in terms of a rapidly convergent infinite power series with easily computable terms.
\end{abstract}

\section{Basic of Homotopy analysis method}

We consider the following differential equation

$$
N[u(\tau)]=0,
$$

where $N$ is a nonlinear operator, $\tau$ denotes independent variable, $u(\tau)$ is an unknown function, respectively. For simplicity, we ignore all boundary or initial conditions, which can be treated in the similar way. By means of generalizing the traditional homotopy method, Liao [7] constructs the so-called zero-order deformation equation

$$
(1-p) L\left[\phi(\tau ; p)-u_{0}(\tau)\right]=\operatorname{phH}(\tau) N[\phi(\tau ; p)]
$$


Where $\mathrm{p} \in[0,1]$ is the embedding parameter, $\mathrm{h} \neq 0$ is a non-zero auxiliary parameter, $H(\tau)$

is an auxiliary function, $L$ is an auxiliary linear operator, $u_{0}(\tau)$ is an initial guess of is an unknown function, respectively. It is important, that one has great freedom to choose auxiliary things in HAM. Obviously, when $p=0$ and $p=1$, it holds

$$
\phi(\tau ; 0)=u_{0}(\tau), \quad \phi(\tau ; 1)=u(\tau)
$$

respectively. Thus, as $p$ increases from 0 to 1 , the solution $\phi(\tau ; p)$ varies from the initial guess $u_{0}(\tau)$ to the solution $u(\tau)$.Expanding $\phi(\tau ; p)$ in Taylor series with respect to $p$, we have

$$
\phi(\tau ; p)=u_{0}(\tau)+\sum_{m=1}^{+\infty} u_{m}(\tau) p^{m}
$$

Where

$$
u_{m}(\tau)=\left.\frac{1}{m !} \frac{\partial^{m} \phi(\tau ; p)}{\partial p^{m}}\right|_{p=0} .
$$

If the auxiliary linear operator, the initial guess, the auxiliary parameter $h$, and the auxiliary function are properly chosen, the series (4) converges at $p=1$, and then we have

$$
u(\tau)=u_{0}(\tau)+\sum_{m=1}^{+\infty} u_{m}(\tau)
$$

which must be one of solutions of original nonlinear equation, as proved by Liao [1]. As $h=-1$ and $H(\tau)=1$, Eq. (2) become

$$
(1-p) L\left[\phi(\tau ; p)-u_{0}(\tau)\right]+p N[\phi(\tau ; p)]=0
$$

Which is used mostly in the homotopy perturbation method, where as the solution obtained directly, without using Taylor series [2, 3]. According to the definition (5), the governing equation can be deduced from the zero-order deformation equation (2).

Define the vector

$$
\vec{u}_{n}=\left\{u_{0}(\tau), u_{1}(\tau), \cdots, u_{n}(\tau)\right\}
$$

Differentiating equation (7) $m$ times with respect to the embedding parameter $p$ and then setting $\mathrm{p}=0$ and finally dividing them by $\mathrm{m}$ !, we have the so-called $\mathrm{m}$ th-order deformation equation 


$$
L\left[u_{m}(\tau)-\chi_{m} u_{m-1}(\tau)\right]=h H(\tau) R_{m}\left(\vec{u}_{m-1}\right)
$$

subject to initial condition

$$
u_{m}(x, 0)=0, \quad \frac{\partial u_{m}(x, 0)}{\partial t}=0,
$$

Where

$$
R_{m}\left(\vec{u}_{m-1}\right)=\left.\frac{1}{(m-1) !} \frac{\partial^{m-1} N[\phi(\tau ; p)]}{\partial p^{m-1}}\right|_{p=0}
$$

And

$$
\chi_{m}= \begin{cases}0 & m \leq 1 \\ 1 & m>1\end{cases}
$$

It should be emphasized that $u_{m}(\tau)$ for $m \geq 1$ is governed by the linear equation (8) with the linear boundary conditions that come from original problem, which can be easily solved by symbolic computation software such as Mathematica. If Eq. (1) admits unique solution, then this method will produce the unique solution. If equation (1) does not possess unique solution, the HAM will give a solution among many other (possible) solutions. For the convergence of the above method we refer the reader to $[5,6,7,9]$.

\section{The Maxwell system in meta materials}

The DNG, metamaterials can be simulated using lossy Drude polarization and magnetization modes. The governing equations for modeling wave propagation in metamaterials are[1,4,10]

$$
\begin{aligned}
& \varepsilon_{0} \frac{\partial E}{\partial t}=\nabla \times H-J \\
& \mu_{0} \frac{\partial H}{\partial t}=-\nabla \times E-K . \\
& \frac{1}{\varepsilon_{0} w_{p e}^{2}} \frac{\partial J}{\partial t}+\frac{\Gamma_{e}}{\varepsilon_{0} w_{p e}^{2}} J=E, \\
& \frac{1}{\mu_{0}{w_{p m}}^{2}} \frac{\partial K}{\partial t}+\frac{\Gamma_{m}}{\mu_{0} w_{p m}^{2}} K=H,
\end{aligned}
$$

where $\varepsilon_{0}$ is the vacuum permittivity, $\mu_{0}$ is the vacuum permeability, $w_{p e}$ and $w_{p m}$ are the electric and magnetic plasma frequencies, respectively, $\Gamma_{e}$ and $\Gamma_{m}$ are the electric and 
magnetic damping frequencies, respectively, $E(x, t)$ and $H(x, t)$ are the electric and magnetic fields, respectively, and $J(x, t)$ and $K(x, t)$ are the induced electric and magnetic currents, respectively. The complex materials make solving the metamaterials model more challenging, since the governing equations cannot be reduced to a simple vector wave equation as in vacuum.

is a For simplicity, we assume that the modeling domain be $\Omega \times(0, T)$, where $\Omega$ bounded Lipschitz polyhedral domain in $R^{3}$ with connected boundary $\partial \Omega_{\text {. }}$ Furthermore, we assume that the boundary of $\Omega$ is perfect conducting so that

$$
n \times E=0 \text { On } \partial \Omega
$$

Where $n$ is the unit outward normal to $\partial \Omega$. Also we assume that the initial conditions are

$$
\begin{aligned}
& E(x, 0)=E_{0}(x), \quad H(x, 0)=H_{0}(x) \\
& J(x, 0)=J_{0}(x), J(x, 0)=J_{0}(x) .
\end{aligned}
$$

Lemma 2.1. There exists a unique solution for system (12)-(15). Furthermore; the solution of the system (12)-(15) satisfies the following stability estimate [4]

$\varepsilon_{0}\|E(t)\|_{0}^{2}+\mu_{0}\|H(t)\|_{0}^{2}+\frac{1}{\mu_{0} w_{p m}^{2}}\|K(t)\|_{0}^{2}+\frac{1}{\varepsilon_{0} w_{p e}{ }^{2}}\|J(t)\|_{0}^{2} \leq \varepsilon_{0}\|E(0)\|_{0}^{2}+$ $\mu_{0}\|H(0)\|_{0}^{2}+\frac{1}{\mu_{0} w_{p m}{ }^{2}}\|K(0)\|_{0}^{2}+\frac{1}{\varepsilon_{0} w_{p e}{ }^{2}}\|J(0)\|_{0}^{2}$.

Lemma 2.2. Assume that the initial conditions are divergence free.i.e.,

$$
\begin{aligned}
& \nabla \cdot\left(\varepsilon_{0} E_{0}\right)=0, \nabla \cdot\left(\mu_{0} H_{0}\right)=0 \\
& \nabla \cdot\left(J_{0}\right)=0, \nabla \cdot\left(K_{0}\right)=0
\end{aligned}
$$

Then for any time $t>0$, the electric field $E$ and electric current $J$ are divergence free. Similary, for any time $t>0$, the magnetic field $H$ and the magnetic current $K$ are divergence free.

\section{The HAM for the Maxwell system in meta materials}

In this section of the paper, we consider the Maxwell system in Meta materials. In system (12)-(15), we carry directly to 2D by using the scalar and vector curl operators

$$
\begin{aligned}
& \nabla \times E=\frac{\partial E_{2}}{\partial x_{1}}-\frac{\partial E_{1}}{\partial x_{2}} \\
& \nabla \times H=H_{z}=\left(\frac{\partial H}{\partial x_{2}},-\frac{\partial H}{\partial x_{1}}\right)^{T}
\end{aligned}
$$


Where the upper index $T$ denotes the vector transpose. Considering the lossy property of metamaterials, we construct the following exact solution for the 2D transverse electrical model (assuming all physical parameters to be one, i.e. $\left(\varepsilon_{0}=\mu_{0}=w_{p e}=\right.$ $\left.w_{p m}=\Gamma_{e}=\Gamma_{m}=1\right)$

$$
\begin{gathered}
E=\left(\begin{array}{l}
E_{x} \\
E_{y}
\end{array}\right)=\left(\begin{array}{c}
-\frac{1}{\sqrt{2}} \cos \pi x \sin \pi y \\
\frac{1}{\sqrt{2}} \sin \pi x \cos \pi y
\end{array}\right) \mathrm{e}^{-\mathrm{t}} \cos \mathrm{t} \\
H=\sqrt{2} \pi(\cos \pi x \cos \pi y) \mathrm{e}^{-\mathrm{t}} \cos \mathrm{t} \\
J=\left(\begin{array}{c}
J_{x} \\
J_{y}
\end{array}\right)=\left(\begin{array}{c}
-\frac{1}{\sqrt{2}} \cos \pi x \sin \pi y \\
\frac{1}{\sqrt{2}} \sin \pi x \cos \pi y
\end{array}\right) \mathrm{e}^{-\mathrm{t}} \sin \mathrm{t} \\
K=\sqrt{2} \pi(\cos \pi x \cos \pi y) \mathrm{e}^{-\mathrm{t}} \sin \mathrm{t}
\end{gathered}
$$

To solve the system. (12-15) by means of homotopy analysis method, we choose the linear operator

$$
\begin{aligned}
& \mathrm{L}_{1}\left[\emptyset_{1}(\mathrm{x}, \mathrm{y}, \mathrm{t} ; \mathrm{p})\right]=\frac{\partial \emptyset_{1}(\mathrm{x}, \mathrm{y}, \mathrm{t} ; \mathrm{p})}{\partial \mathrm{t}} \\
& \mathrm{L}_{2}\left[\emptyset_{2}(\mathrm{x}, \mathrm{y}, \mathrm{t} ; \mathrm{p})\right]=\frac{\partial \emptyset_{2}(\mathrm{x}, \mathrm{y}, \mathrm{t} ; \mathrm{p})}{\partial \mathrm{t}} \\
& \mathrm{L}_{3}\left[\emptyset_{3}(\mathrm{x}, \mathrm{y}, \mathrm{t} ; \mathrm{p})\right]=\frac{\partial \emptyset_{3}(\mathrm{x}, \mathrm{y}, \mathrm{t} ; \mathrm{p})}{\partial \mathrm{t}} \\
& \mathrm{L}_{4}\left[\emptyset_{4}(\mathrm{x}, \mathrm{y}, \mathrm{t} ; \mathrm{p})\right]=\frac{\partial \emptyset_{4}(\mathrm{x}, \mathrm{y}, \mathrm{t} ; \mathrm{p})}{\partial \mathrm{t}} \\
& \mathrm{L}_{5}\left[\emptyset_{5}(\mathrm{x}, \mathrm{y}, \mathrm{t} ; \mathrm{p})\right]=\frac{\partial \emptyset_{5}(\mathrm{x}, \mathrm{y}, \mathrm{t} ; \mathrm{p})}{\partial \mathrm{t}} \\
& \mathrm{L}_{6}\left[\emptyset_{6}(\mathrm{x}, \mathrm{y}, \mathrm{t} ; \mathrm{p})\right]=\frac{\partial \emptyset_{6}(\mathrm{x}, \mathrm{y}, \mathrm{t} ; \mathrm{p})}{\partial \mathrm{t}}
\end{aligned}
$$

With the property: $L_{i}\left[c_{i}\right]=0 i=1,2,3,4,5,6$

where $c_{i}$ are integral constants. The inverse $\mathrm{L}_{\mathrm{i}}^{-1}$ operator are given by 


$$
\mathrm{L}_{\mathrm{i}}^{-1}=\int_{0}^{\mathrm{t}}(.) \mathrm{dt}
$$

We now define a nonlinear operator as

$$
\begin{gathered}
N_{1}\left[\emptyset_{1}, \emptyset_{2}, \emptyset_{3}, \emptyset_{4}, \emptyset_{5}, \emptyset_{6}\right]=\varepsilon_{0} \frac{\partial \emptyset_{1}}{\partial \mathrm{t}}-\frac{\partial \emptyset_{3}}{\partial \mathrm{y}}+\emptyset_{4} \\
N_{2}\left[\emptyset_{1}, \emptyset_{2}, \emptyset_{3}, \emptyset_{4}, \emptyset_{5}, \emptyset_{6}\right]=\varepsilon_{0} \frac{\partial \emptyset_{2}}{\partial \mathrm{t}}+\frac{\partial \emptyset_{3}}{\partial \mathrm{x}}+\emptyset_{5} \\
N_{3}\left[\emptyset_{1}, \emptyset_{2}, \emptyset_{3}, \emptyset_{4}, \emptyset_{5}, \emptyset_{6}\right]=\mu_{0} \frac{\partial \emptyset_{3}}{\partial \mathrm{t}}+\frac{\partial \emptyset_{2}}{\partial \mathrm{x}}-\frac{\partial \emptyset_{1}}{\partial \mathrm{y}}-\emptyset_{6} \\
N_{4}\left[\emptyset_{1}, \emptyset_{2}, \emptyset_{3}, \emptyset_{4}, \emptyset_{5}, \emptyset_{6}\right]=\frac{1}{\varepsilon_{0} w_{p e}{ }^{2}} \frac{\partial \emptyset_{4}}{\partial \mathrm{t}}+\frac{\Gamma_{e}}{\varepsilon_{0} w_{p e}{ }^{2}} \emptyset_{4}-\emptyset_{1} \quad(24) \\
N_{5}\left[\emptyset_{1}, \emptyset_{2}, \emptyset_{3}, \emptyset_{4}, \emptyset_{5}, \emptyset_{6}\right]=\frac{1}{\varepsilon_{0} w_{p e}^{2}} \frac{\partial \emptyset_{5}}{\partial \mathrm{t}}+\frac{\Gamma_{e}}{\varepsilon_{0} w_{p e}^{2}} \emptyset_{5}-\emptyset_{2} \\
N_{6}\left[\emptyset_{1}, \emptyset_{2}, \emptyset_{3}, \emptyset_{4}, \emptyset_{5}, \emptyset_{6}\right]=\frac{1}{\mu_{0} w_{p m}{ }^{2}} \frac{\partial \emptyset_{6}}{\partial \mathrm{t}}+\frac{\Gamma_{m}}{\mu_{0} w_{p m}^{2}} \emptyset_{6}-\emptyset_{3}
\end{gathered}
$$

Using above definition, we construct the zeroth-order deformation equation

$$
\begin{aligned}
& (1-\mathrm{p}) \mathrm{L}_{1}\left[\emptyset_{1}(\mathrm{x}, \mathrm{y}, \mathrm{t} ; \mathrm{p})-\mathrm{E}_{\mathrm{x} 0}(\mathrm{x}, \mathrm{y}, \mathrm{t})\right]=p h_{1} N_{1}\left[\emptyset_{1}, \emptyset_{2}, \emptyset_{3}, \emptyset_{4}, \emptyset_{5}, \emptyset_{6}\right] \\
& (1-\mathrm{p}) \mathrm{L}_{2}\left[\emptyset_{2}(\mathrm{x}, \mathrm{y}, \mathrm{t} ; \mathrm{p})-\mathrm{E}_{\mathrm{y}_{0}}(\mathrm{x}, \mathrm{y}, \mathrm{t})\right]=p h_{2} N_{2}\left[\emptyset_{1}, \emptyset_{2}, \emptyset_{3}, \emptyset_{4}, \emptyset_{5}, \emptyset_{6}\right] \\
& (1-\mathrm{p}) \mathrm{L}_{3}\left[\emptyset_{3}(\mathrm{x}, \mathrm{y}, \mathrm{t} ; \mathrm{p})-\mathrm{H}_{0}(\mathrm{x}, \mathrm{y}, \mathrm{t})\right]=p h_{3} N_{3}\left[\emptyset_{1}, \emptyset_{2}, \emptyset_{3}, \emptyset_{4}, \emptyset_{5}, \emptyset_{6}\right] \\
& (1-\mathrm{p}) \mathrm{L}_{4}\left[\emptyset_{4}(\mathrm{x}, \mathrm{y}, \mathrm{t} ; \mathrm{p})-\mathrm{J}_{\mathrm{x} 0}(\mathrm{x}, \mathrm{y}, \mathrm{t})\right]=p h_{4} N_{4}\left[\emptyset_{1}, \emptyset_{2}, \emptyset_{3}, \emptyset_{4}, \emptyset_{5}, \emptyset_{6}\right] \\
& (1-\mathrm{p}) \mathrm{L}_{5}\left[\emptyset_{5}(\mathrm{x}, \mathrm{y}, \mathrm{t} ; \mathrm{p})-\mathrm{J}_{\mathrm{y}_{0}}(\mathrm{x}, \mathrm{y}, \mathrm{t})\right]=p h_{5} N_{5}\left[\emptyset_{1}, \emptyset_{2}, \emptyset_{3}, \emptyset_{4}, \emptyset_{5}, \emptyset_{6}\right] \\
& (1-\mathrm{p}) \mathrm{L}_{6}\left[\emptyset_{6}(\mathrm{x}, \mathrm{y}, \mathrm{t} ; \mathrm{p})-\mathrm{K}_{0}(\mathrm{x}, \mathrm{y}, \mathrm{t})\right]=p h_{6} N_{6}\left[\emptyset_{1}, \emptyset_{2}, \emptyset_{3}, \emptyset_{4}, \emptyset_{5}, \emptyset_{6}\right]
\end{aligned}
$$

With the initial condition:

$$
\begin{array}{ll}
\emptyset_{1}(\mathrm{x}, \mathrm{y}, \mathrm{t} ; 0)=\mathrm{E}_{\mathrm{x} 0}(\mathrm{x}, \mathrm{y}, \mathrm{t}) \cdot & \emptyset_{1}(\mathrm{x}, \mathrm{y}, \mathrm{t} ; 1)=\mathrm{E}_{\mathrm{x}}(\mathrm{x}, \mathrm{y}, \mathrm{t}) \\
\emptyset_{2}(\mathrm{x}, \mathrm{y}, \mathrm{t} ; 0)=\mathrm{E}_{\mathrm{y}_{0}}(\mathrm{x}, \mathrm{y}, \mathrm{t}) \cdot & \emptyset_{2}(\mathrm{x}, \mathrm{y}, \mathrm{t} ; 1)=\mathrm{E}_{\mathrm{y}}(\mathrm{x}, \mathrm{y}, \mathrm{t}) \\
\emptyset_{3}(\mathrm{x}, \mathrm{y}, \mathrm{t} ; 0)=H_{0}(\mathrm{x}, \mathrm{y}, \mathrm{t}) \cdot & \emptyset_{3}(\mathrm{x}, \mathrm{y}, \mathrm{t} ; 1)=H(\mathrm{x}, \mathrm{y}, \mathrm{t})
\end{array}
$$




$$
\begin{array}{ll}
\emptyset_{4}(\mathrm{x}, \mathrm{y}, \mathrm{t} ; 0)=\mathrm{J}_{\mathrm{x}}(\mathrm{x}, \mathrm{y}, \mathrm{t}) & \emptyset_{4}(\mathrm{x}, \mathrm{y}, \mathrm{t} ; 1)=\mathrm{J}_{\mathrm{x}}(\mathrm{x}, \mathrm{y}, \mathrm{t}) \\
\emptyset_{5}(\mathrm{x}, \mathrm{y}, \mathrm{t} ; 0)=\mathrm{J}_{\mathrm{y}}(\mathrm{x}, \mathrm{y}, \mathrm{t}) & \emptyset_{5}(\mathrm{x}, \mathrm{y}, \mathrm{t} ; 1)=\mathrm{J}_{\mathrm{y}}(\mathrm{x}, \mathrm{y}, \mathrm{t}) \\
\emptyset(\mathrm{x}, \mathrm{y}, \mathrm{t} ; 0)=\mathrm{K}(\mathrm{x}, \mathrm{y}, \mathrm{t}) & \emptyset(\mathrm{x}, \mathrm{y}, \mathrm{t} ; 1)=\mathrm{K}(\mathrm{x}, \mathrm{y}, \mathrm{t})
\end{array}
$$

Thus, we obtain the $m$ th-order deformation equations

$$
\begin{aligned}
& \mathrm{L}_{1}\left[\mathrm{E}_{\mathrm{x} \mathrm{m}}(\mathrm{x}, \mathrm{y}, \mathrm{t})-\chi_{m} \mathrm{E}_{\mathrm{x} m-1}(\mathrm{x}, \mathrm{y}, \mathrm{t})\right]=h_{1} R_{1_{m}}\left[\overrightarrow{\mathrm{E}}_{\mathrm{x}-1}, \overrightarrow{\mathrm{E}}_{\mathrm{m}-1}, \vec{H}_{\mathrm{m}-1},{\overrightarrow{\mathrm{J}_{\mathrm{m}}-1}}_{\mathrm{J}_{\mathrm{y}}-1}, \vec{K}_{\mathrm{m}-1}\right] \\
& \mathrm{L}_{2}\left[\mathrm{E}_{\mathrm{y}_{\mathrm{m}}}(\mathrm{x}, \mathrm{y}, \mathrm{t})-\chi_{m} \mathrm{E}_{\mathrm{y}_{\mathrm{m}-1}}(\mathrm{x}, \mathrm{y}, \mathrm{t})\right]=h_{2} R_{2 m}\left[\overrightarrow{\mathrm{E}}_{\mathrm{x}-1}, \overrightarrow{\mathrm{E}}_{\mathrm{y}_{\mathrm{m}-1}}, \vec{H}_{\mathrm{m}-1},{\overrightarrow{\mathrm{J}_{\mathrm{m}}-1}}_{\mathrm{J}_{\mathrm{y}}-1}, \vec{K}_{\mathrm{m}-1}\right] \\
& \mathrm{L}_{3}\left[\mathrm{H}_{\mathrm{m}}(\mathrm{x}, \mathrm{y}, \mathrm{t})-\chi_{m} \mathrm{H}_{\mathrm{m}-1}(\mathrm{x}, \mathrm{y}, \mathrm{t})\right]=h_{3} R_{3 \mathrm{~m}}\left[\overrightarrow{\mathrm{E}}_{\mathrm{x}-1}, \overrightarrow{\mathrm{E}}_{\mathrm{y}-1}, \vec{H}_{\mathrm{m}-1}, \overrightarrow{\mathrm{j}}_{\mathrm{x}-1}, \overrightarrow{\mathrm{y}}_{\mathrm{m}-1}, \vec{K}_{\mathrm{m}-1}\right] \\
& \mathrm{L}_{4}\left[\mathrm{~J}_{\mathrm{x}_{\mathrm{m}}}(\mathrm{x}, \mathrm{y}, \mathrm{t})-\chi_{m} \mathrm{~J}_{\mathrm{x}_{\mathrm{m}-1}}(\mathrm{x}, \mathrm{y}, \mathrm{t})\right]=h_{5} R_{4 m}\left[\overrightarrow{\mathrm{E}}_{\mathrm{x}-1}, \overrightarrow{\mathrm{E}}_{\mathrm{m}-1}, \vec{H}_{\mathrm{m}-1}, \overrightarrow{\mathrm{J}}_{\mathrm{x}-1}, \overrightarrow{\mathrm{J}}_{\mathrm{m}-1}, \vec{K}_{\mathrm{m}-1}\right] \\
& \mathrm{L}_{5}\left[\mathrm{~J}_{\mathrm{y}_{\mathrm{m}}}(\mathrm{x}, \mathrm{y}, \mathrm{t})-\chi_{m} \mathrm{~J}_{\mathrm{y}_{\mathrm{m}-1}}(\mathrm{x}, \mathrm{y}, \mathrm{t})\right]=h_{5} R_{5 \mathrm{~m}}\left[\overrightarrow{\mathrm{E}}_{\mathrm{x}-1}, \overrightarrow{\mathrm{E}}_{\mathrm{m}-1}, \vec{H}_{\mathrm{m}-1}, \overrightarrow{\mathrm{j}}_{\mathrm{x}-1}, \overrightarrow{\mathrm{J}}_{\mathrm{m}-1}, \vec{K}_{\mathrm{m}-1}\right] \\
& \mathrm{L}_{6}\left[\mathrm{H}_{\mathrm{m}}(\mathrm{x}, \mathrm{y}, \mathrm{t})-\chi_{m} \mathrm{H}_{\mathrm{m}-1}(\mathrm{x}, \mathrm{y}, \mathrm{t})\right]=h_{6} R_{6_{\mathrm{m}}}\left[\overrightarrow{\mathrm{E}}_{\mathrm{x}_{\mathrm{m}-1}}, \overrightarrow{\mathrm{E}}_{\mathrm{m}-1}, \vec{H}_{\mathrm{m}-1}, \overrightarrow{\mathrm{j}}_{\mathrm{m}-1}, \overrightarrow{\mathrm{J}}_{\mathrm{m}-1}, \vec{K}_{\mathrm{m}-1}\right]
\end{aligned}
$$

where

$$
\begin{aligned}
& R_{1_{m}}\left[\overrightarrow{\mathrm{E}}_{\mathrm{x}_{\mathrm{m}-1}}, \overrightarrow{\mathrm{E}}_{\mathrm{y}_{\mathrm{m}-1}}, \vec{H}_{\mathrm{m}-1}, \overrightarrow{\mathrm{j}}_{\mathrm{m}-1}, \overrightarrow{\mathrm{J}}_{\mathrm{m}-1}, \vec{K}_{\mathrm{m}-1}\right]=\varepsilon_{0} \frac{\partial \mathrm{E}_{\mathrm{x}_{\mathrm{m}}-1}}{\partial \mathrm{t}}-\frac{\partial \mathrm{H}_{\mathrm{m}-1}}{\partial \mathrm{y}}+\mathrm{J}_{\mathrm{x}_{\mathrm{m}-1}} \\
& R_{2 m}\left[\overrightarrow{\mathrm{E}}_{\mathrm{x}-1}, \overrightarrow{\mathrm{E}}_{\mathrm{m}-1}, \vec{H}_{\mathrm{m}-1}, \overrightarrow{\mathrm{j}}_{\mathrm{m}-1},{\overrightarrow{\mathrm{y}_{\mathrm{m}}-1}}, \vec{K}_{\mathrm{m}-1}\right]=\varepsilon_{0} \frac{\partial \mathrm{E}_{\mathrm{y}_{\mathrm{m}}-1}}{\partial \mathrm{t}}+\frac{\partial \mathrm{H}_{\mathrm{m}-1}}{\partial \mathrm{x}}+\mathrm{J}_{\mathrm{y}_{\mathrm{m}-1}} \\
& R_{3 m}\left[\overrightarrow{\mathrm{E}}_{\mathrm{m}-1},{\overrightarrow{\mathrm{E}_{\mathrm{y}}-1}}, \vec{H}_{\mathrm{m}-1}, \overrightarrow{\mathrm{J}}_{\mathrm{m}-1}, \overrightarrow{\mathrm{J}}_{\mathrm{m}-1}, \vec{K}_{\mathrm{m}-1}\right]=\mu_{0} \frac{\partial \mathrm{H}_{\mathrm{m}-1}}{\partial \mathrm{t}}+\frac{\partial \mathrm{E}_{\mathrm{y}_{\mathrm{m}}-1}}{\partial \mathrm{x}}-\frac{\partial \mathrm{E}_{\mathrm{x}_{\mathrm{m}}-1}}{\partial \mathrm{y}}-K_{\mathrm{m}-1} \\
& R_{4 m}\left[{\overrightarrow{\mathrm{E}_{\mathrm{m}}-1}}, \overrightarrow{\mathrm{E}}_{\mathrm{y}-1}, \vec{H}_{\mathrm{m}-1}, \overrightarrow{\mathrm{J}}_{\mathrm{m}-1}, \overrightarrow{\mathrm{J}}_{\mathrm{m}-1}, \vec{K}_{\mathrm{m}-1}\right]=\frac{1}{\varepsilon_{0} w_{p e}{ }^{2}} \frac{\partial \mathrm{J}_{\mathrm{x}_{\mathrm{m}}-1}}{\partial \mathrm{t}}+\frac{\Gamma_{e}}{\varepsilon_{0} w_{p e}{ }^{2}} \mathrm{~J}_{\mathrm{x}_{\mathrm{m}-1}}-\mathrm{E}_{\mathrm{x}_{\mathrm{m}-1}} \\
& R_{5 m}\left[\overrightarrow{\mathrm{E}}_{\mathrm{x} m-1},{\overrightarrow{\mathrm{E}_{\mathrm{m}}-1}}, \vec{H}_{\mathrm{m}-1},{\overrightarrow{\mathrm{x}_{\mathrm{m}}-1}}_{\mathrm{J}_{\mathrm{y}-1}}, \vec{K}_{\mathrm{m}-1}\right]=\frac{1}{\varepsilon_{0} w_{p e}{ }^{2}} \frac{\partial \mathrm{J}_{\mathrm{y}_{\mathrm{m}}-1}}{\partial \mathrm{t}}+\frac{\Gamma_{e}}{\varepsilon_{0} w_{p e}{ }^{2}} \mathrm{~J}_{\mathrm{x}-1}-\mathrm{E}_{\mathrm{y}_{\mathrm{m}-1}} \\
& R_{6_{m}}\left[\overline{\mathrm{E}}_{\mathrm{x}_{\mathrm{m}-1}}, \overline{\mathrm{E}}_{\mathrm{y}_{\mathrm{m}-1}}, \bar{H}_{\mathrm{m}-1}, \overline{\mathrm{J}}_{\mathrm{x}_{\mathrm{m}-1}}, \overline{\mathrm{J}}_{\mathrm{y}_{\mathrm{m}-1}}, \bar{K}_{\mathrm{m}-1}\right]=\frac{1}{\mu_{0} w_{p m}} \frac{\partial K_{\mathrm{m}-1}}{\partial \mathrm{t}}+\frac{\Gamma_{m}}{\mu_{0} w_{p m}^{2}} K_{\mathrm{m}-1}-\mathrm{H}_{\mathrm{m}-1}
\end{aligned}
$$

Now the solution of the $m$-th-order deformation Eq. (26) for $m \geq 1$

$$
\begin{aligned}
& \mathrm{E}_{\mathrm{x}}(\mathrm{x}, \mathrm{y}, \mathrm{t})=\chi_{m} \mathrm{E}_{\mathrm{x}_{\mathrm{m}-1}}(\mathrm{x}, \mathrm{y}, \mathrm{t})+h_{1} L^{-1}\left[R_{1_{m}}\left[\overrightarrow{\mathrm{E}}_{\mathrm{m}-1},{\overrightarrow{\mathrm{E}_{\mathrm{m}}-1}}_{H_{\mathrm{m}-1}}, \overrightarrow{\mathrm{J}}_{\mathrm{m}-1}, \overrightarrow{\mathrm{J}}_{\mathrm{m}-1}, \vec{K}_{\mathrm{m}-1}\right]\right. \\
& \mathrm{E}_{\mathrm{y}_{\mathrm{m}}}(\mathrm{x}, \mathrm{y}, \mathrm{t})=\chi_{m} \mathrm{E}_{\mathrm{x}_{\mathrm{m}-1}}(\mathrm{x}, \mathrm{y}, \mathrm{t})+h_{2} L^{-1}\left[R_{2 m}\left[\overrightarrow{\mathrm{E}}_{\mathrm{x}_{\mathrm{m}}-1}, \overrightarrow{\mathrm{E}}_{\mathrm{y}_{\mathrm{m}-1}}, \vec{H}_{\mathrm{m}-1}, \overrightarrow{\mathrm{J}}_{\mathrm{m}-1}, \overrightarrow{\mathrm{J}}_{\mathrm{m}-1}, \vec{K}_{\mathrm{m}-1}\right]\right.
\end{aligned}
$$




$$
\begin{aligned}
& \mathrm{H}_{\mathrm{m}}(\mathrm{x}, \mathrm{y}, \mathrm{t})=\chi_{m} \mathrm{H}_{\mathrm{m}-1}(\mathrm{x}, \mathrm{y}, \mathrm{t})+h_{3} L^{-1}\left[R_{3 m}\left[\overrightarrow{\mathrm{E}}_{\mathrm{x}-1},{\overrightarrow{\mathrm{E}_{\mathrm{y}}-1}}, \vec{H}_{\mathrm{m}-1}, \overrightarrow{\mathrm{J}}_{\mathrm{x}-1}, \overrightarrow{\mathrm{J}}_{\mathrm{m}-1}, \vec{K}_{\mathrm{m}-1}\right]\right. \\
& \mathrm{J}_{\mathrm{x}}(\mathrm{x}, \mathrm{y}, \mathrm{t})=\chi_{m} \mathrm{~J}_{\mathrm{x}_{\mathrm{m}-1}}(\mathrm{x}, \mathrm{y}, \mathrm{t})+h_{4} L^{-1}\left[R_{4_{m}}\left[{\overrightarrow{\mathrm{E}_{\mathrm{x}}-1}},{\overrightarrow{\mathrm{E}_{\mathrm{y}}-1}}, \vec{H}_{\mathrm{m}-1}, \overrightarrow{\mathrm{J}}_{\mathrm{x}-1}, \overrightarrow{\mathrm{J}}_{\mathrm{m}-1}, \vec{K}_{\mathrm{m}-1}\right]\right. \\
& \mathrm{J}_{\mathrm{y}}(\mathrm{x}, \mathrm{y}, \mathrm{t})=\chi_{m} \mathrm{~J}_{\mathrm{x}_{\mathrm{m}-1}}(\mathrm{x}, \mathrm{y}, \mathrm{t})+h_{5} L^{-1}\left[R_{5 \mathrm{~m}}\left[\overrightarrow{\mathrm{E}}_{\mathrm{x}_{\mathrm{m}-1}}, \overrightarrow{\mathrm{E}}_{\mathrm{y}_{\mathrm{m}-1}}, \vec{H}_{\mathrm{m}-1},{\overrightarrow{\mathrm{J}_{\mathrm{x}}-1}}_{\mathrm{J}_{\mathrm{y}}-1}, \vec{K}_{\mathrm{m}-1}\right]\right. \\
& \mathrm{K}_{\mathrm{m}}(\mathrm{x}, \mathrm{y}, \mathrm{t})=\chi_{m} \mathrm{~K}_{\mathrm{m}-1}(\mathrm{x}, \mathrm{y}, \mathrm{t})+h_{6} L^{-1}\left[R_{3 \mathrm{~m}}\left[{\overrightarrow{\mathrm{E}_{\mathrm{m}}-1}}_{\mathrm{E}_{\mathrm{y}}-1}, \vec{H}_{\mathrm{m}-1}, \overrightarrow{\mathrm{j}}_{\mathrm{m}-1}, \overrightarrow{\mathrm{J}}_{\mathrm{m}-1}, \vec{K}_{\mathrm{m}-1}\right]\right.
\end{aligned}
$$

We start with under initial approximations:

$$
\begin{gathered}
\mathrm{E}_{0}=\left(\begin{array}{l}
E_{x} \\
E_{y}
\end{array}\right)=\left(\begin{array}{c}
-\frac{1}{\sqrt{2}} \cos \pi x \sin \pi y \\
\frac{1}{\sqrt{2}} \sin \pi x \cos \pi y
\end{array}\right) \\
\mathrm{H}_{0}=\sqrt{2} \pi(\cos \pi x \cos \pi y) \mathrm{e}^{-\mathrm{t}} \cos \mathrm{t} \\
J_{0}=\left(\begin{array}{l}
J_{x} \\
J_{y}
\end{array}\right)=\left(\begin{array}{c}
-\frac{1}{\sqrt{2}} \cos \pi x \sin \pi y \\
\frac{1}{\sqrt{2}} \sin \pi x \cos \pi y
\end{array}\right) \mathrm{e}^{-\mathrm{t}} \sin \mathrm{t} \\
\mathrm{K}_{0}=\sqrt{2} \pi(\cos \pi x \cos \pi y) \mathrm{e}^{-\mathrm{t}} \sin \mathrm{t}
\end{gathered}
$$

By means of the above iteration formula (28) if $h_{i}=-1$, we can obtain directly the other components as

$$
\begin{aligned}
& \mathrm{E}(\mathrm{x}, \mathrm{y}, \mathrm{t})=\left[\begin{array}{l}
\mathrm{E}_{\mathrm{x}} \\
\mathrm{E}_{\mathrm{y}}
\end{array}\right]=\left[\begin{array}{c}
\mathrm{E}_{\mathrm{x}_{0}}(\mathrm{x}, \mathrm{y}, \mathrm{t})+\sum_{\mathrm{i}=0}^{+\infty} \mathrm{E}_{\mathrm{x}_{i}}(\mathrm{x}, \mathrm{y}, \mathrm{t}) \\
E_{\mathrm{y}_{0}}(\mathrm{x}, \mathrm{y}, \mathrm{t})+\sum_{\mathrm{i}=0}^{+\infty} E_{\mathrm{y}_{\mathrm{i}}}(\mathrm{x}, \mathrm{y}, \mathrm{t})
\end{array}\right]= \\
& {\left[\begin{array}{c}
\frac{\cos (\pi x) \sin (\pi y) e^{-t}\left(-\cos (t)+4 \pi^{2} e^{t} t h_{1}+2 \pi^{2} e^{t} t \varepsilon h_{1}{ }^{2}\right.}{\sqrt{2}}+\cdots \\
-\frac{\cos (\pi y) \sin (\pi x) e^{-t}\left(-\cos (t)+4 \pi^{2} e^{t} t h_{2}+2 \pi^{2} e^{t} t \varepsilon h_{2}{ }^{2}\right.}{\sqrt{2}}+\cdots
\end{array}\right]}
\end{aligned}
$$

$$
\begin{aligned}
H(x, y, t)= & H_{0}(x, y, t) \\
& +\sum_{i=0}^{+\infty} H_{i}(x, y, t) \\
& =\sqrt{2} e^{-t} \cos (\pi x) \cos (\pi y)\left(\cos (t)+2 e^{t} t h_{3}+e^{t} t \mu h_{3}{ }^{2}+\cdots\right.
\end{aligned}
$$




$$
\begin{aligned}
& J(x, y, t)=\left[\begin{array}{l}
\mathrm{J}_{\mathrm{x}} \\
J_{\mathrm{y}}
\end{array}\right]=\left[\begin{array}{l}
\mathrm{J}_{\mathrm{x}_{0}}(\mathrm{x}, \mathrm{y}, \mathrm{t})+\sum_{\mathrm{i}=0}^{+\infty} \mathrm{J}_{\mathrm{x}_{i}}(\mathrm{x}, \mathrm{y}, \mathrm{t}) \\
J_{\mathrm{y}_{0}}(\mathrm{x}, \mathrm{y}, \mathrm{t})+\sum_{\mathrm{i}=0}^{+\infty} J_{\mathrm{y}_{\mathrm{i}}}(\mathrm{x}, \mathrm{y}, \mathrm{t})
\end{array}\right]= \\
& {\left[\begin{array}{l}
\frac{e^{-t} \mathrm{t} \cos (\pi x) \sin (\pi y) h_{4}\left(-2 \varepsilon w_{p e}{ }^{2} \sin (t)+2 e^{t} t h_{4}{ }^{2}+e^{t} t^{2} \varepsilon h_{4} w_{p e}{ }^{2}+e^{t} t^{2} h_{4}{ }^{2} \Gamma_{e}\right)}{2 \varepsilon w_{p e}{ }^{2} \sqrt{2}}+\cdots \\
\frac{e^{-t} \mathrm{t} \cos (\pi x) \sin (\pi x) h_{5}\left(-2 \varepsilon w_{p e}{ }^{2} \sin (t)+2 e^{t} t h_{5}{ }^{2}+e^{t} t^{2} \varepsilon h_{5} w_{p e}{ }^{2}+e^{t} t^{2} h_{5}{ }^{2} \Gamma_{e}\right)}{2 \varepsilon w_{p e}{ }^{2} \sqrt{2}}+\cdots
\end{array}\right]}
\end{aligned}
$$

$K(\mathrm{x}, \mathrm{y}, \mathrm{t})$

$=\mathrm{K}_{0}(\mathrm{x}, \mathrm{y}, \mathrm{t})$

$+\sum_{\mathrm{i}=0}^{+\infty} \mathrm{K}_{\mathrm{i}}(\mathrm{x}, \mathrm{y}, \mathrm{t})$

$=-\frac{\pi e^{-t} \mathrm{t} \cos (\pi x) \cos (\pi y) h_{6}\left(-2 \sin (t) \mu w_{p m}{ }^{2}+2 e^{t} t h_{6}{ }^{2}+e^{-t} t^{2} \Gamma_{m} h_{6}{ }^{2}+e^{t} t^{2} \mu h_{6} w_{p m}{ }^{2}\right.}{\mu \sqrt{2} w_{p m}{ }^{2}}$ $+\cdots$

We now choose: $\varepsilon=-1, \mu=-1, m=5, h_{i}=-1, w_{p m}=1, w_{p e}=1, \Gamma_{e}=1, \Gamma_{m}=1$, and then obtain:

$$
\begin{aligned}
& \mathbf{E}(\mathrm{x}, \mathrm{y}, \mathrm{t})=\left[\begin{array}{c}
\frac{\cos (\pi x) \sin (\pi y)\left(-1+4 \pi^{2} t h_{1}+2 \pi^{2} t \varepsilon h_{1}{ }^{2}\right.}{\sqrt{2}} \\
-\frac{\cos (\pi y) \sin (\pi x)\left(-1+4 \pi^{2} t h_{2}+2 \pi^{2} t \varepsilon h_{2}{ }^{2}\right.}{\sqrt{2}}
\end{array}\right] \\
& \mathrm{H}(\mathrm{x}, \mathrm{y}, \mathrm{t})=\sqrt{2} \cos (\pi x) \cos (\pi y)\left(1+2 t h_{3}+t \mu{h_{3}}^{2}\right) \\
& J(x, y, t)=\left[\begin{array}{l}
\frac{\mathrm{t} \cos (\pi x) \sin (\pi y) h_{4}\left(4 \varepsilon w_{p e}{ }^{2}+2 h_{4}+t h_{4} \Gamma_{e}\right.}{2 \varepsilon w_{p e}{ }^{2} \sqrt{2}} \\
\frac{\mathrm{t} \cos (\pi x) \sin (\pi x) h_{5}\left(4 \varepsilon w_{p e}{ }^{2}+2 h_{5}+t h_{5} \Gamma_{e}\right.}{2 \varepsilon w_{p e}{ }^{2} \sqrt{2}}
\end{array}\right] \\
& K(\mathrm{x}, \mathrm{y}, \mathrm{t})=-\begin{array}{c}
\pi \mathrm{t} \cos (\pi x) \cos (\pi y) h_{6}\left(4 \mu w_{p m}{ }^{2}+2 h_{6}+t \Gamma_{m} h_{6}\right. \\
\mu \sqrt{2} 2 w
\end{array}
\end{aligned}
$$

And then choose: $\varepsilon=-2, \mu=-2, m=5, h_{i}=-1, w_{p m}=1, w_{p e}=1, \Gamma_{e}=1, \Gamma_{m}=1$, with this chose, we have: 


$$
\begin{aligned}
& \mathrm{E}(\mathrm{x}, \mathrm{y}, \mathrm{t})=\left[\begin{array}{c}
-\frac{\cos (\pi x) \sin (\pi y)\left(1+80 \pi^{2} t\right)}{\sqrt{2}} \\
\frac{\cos (\pi y) \sin (\pi x)\left(1+80 \pi^{2} t\right)}{\sqrt{2}}
\end{array}\right] \\
& \mathrm{H}(\mathrm{x}, \mathrm{y}, \mathrm{t})=\sqrt{2} \pi \cos (\pi x) \cos (\pi y)(-1+40 t) \\
& J(x, y, t)=\left[\begin{array}{c}
-\frac{\mathrm{t} \cos (\pi x) \sin (\pi y) t\left(1560+516 t+44 t^{2}+t^{3}\right)}{192 \sqrt{2}} \\
\frac{\mathrm{t} \cos (\pi y) \sin (\pi x) t\left(1560+516 t+44 t^{2}+t^{3}\right)}{192 \sqrt{2}}
\end{array}\right] \\
& K(\mathrm{x}, \mathrm{y}, \mathrm{t})=\frac{\pi \mathrm{t} \cos (\pi x) \cos (\pi y)\left(1560+516 t+44 t^{2}+t^{3}\right)}{96 \sqrt{2}}
\end{aligned}
$$

\section{Conclusion}

Homotopy analysis method is a powerful method which yields a convergent series solution for Maxwell's equation system. This method is better than numerical methods, as it is free from rounding off errors, and does not require large computer power. It is apparently seen that HAM is a very powerful and efficient technique in finding analytical solutions for wide classes of linear and nonlinear problems. The results show that HAM is a powerful mathematical tool for solving nonlinear partial differential equations and systems of nonlinear partial differential equations.

Mathematica has been used for computations in this article.

\section{References}

[1] Adomian G., Coupled Maxwell Equations for Electromagnetic Scattering, Applied Mathemathics,And Compution, 77(1996)133-135.

[2] He JH. Homotopy perturbation method for solving boundary value problems, Phys Lett A,350(12) (2006)87-88.

[3] He JH. Some asymptotic methods for strongly nonlinear equations, Int J Mod Phys

$$
\text { B, 20(10) (2006) 1141-1199. }
$$

[4] Jichun Li, Numerical convergence and physical fidelity analysis for Maxwell's equations in metamaterials,comput.Methods Appl.Mech.Engrg.198(2009)3161-3172.

[5] Jafari H., Saeidy M., Firoozjaee M. A., The Homotopy Analysis Method for Solving Higher Dimensional Initial Boundary Value Problems of Variable Coefficients, Numerical Methods for Partial Differential Equations, Wiley Periodicals, Inc. Numer Methods Partial Differential Eq 26(2010)1021-1032. 
A. Zare, M.A. Firoozjaee / TJMCS Vol .3 No.2 (2011) 225 - 235

[6] Jafari H., Firoozjaee M. A. Multistage Homotopy Analysis Method for Solving Nonlinear Integral Equations, Applications and Applied Mathematic International Journal

(AAM)1 (2010)34-35.

[7] Liao SJ. Beyond perturbation: introduction to the homotopy analysis method. CRC Press, Boca Raton:Chapman \& Hall; 2003.

[8] Liao SJ. The proposed homotopy analysis technique for the solution of nonlinear problems, Ph.D. Thesis,Shanghai Jiao Tong University, 1992.

[9] S.J. Liao, On the homotopy analysis method for nonlinear problems, Appl Math Comput, 147 (2004), 499-513.

[10] Victorita Dolean, Stephane Lanteri ,Ronan Perrussel, A domain decomposition method for solving thethree-dimensional time-harmonic Maxwell equations discretized by discontinuous Galerkin methods, Journal of Computational Physics 227 (2008) 2044-2072. 